\title{
Charm Reflects Poorly on Anticharm
}

\author{
A study of particles containing charm quarks has uncovered a violation of so-called \\ $C P$ symmetry, which could help in understanding why matter dominates antimatter in the \\ Universe.
}

\section{by Hiroaki Aihara}

$\mathrm{T}$ he Universe is not symmetric with respect to matter and antimatter, as evidenced by the dominance of the former over the latter. For example, we see hydrogen everywhere in nature but can only make antihydrogen in the lab with great effort. The origin of this lopsidedness in the Universe has eluded theorists, but it is thought to be related to violations of charge-parity $(C P)$ symmetry. This symmetry, which says that an elementary particle behaves like a mirror reflection of its antimatter partner, is mostly respected within the standard model of elementary particle physics, but it is violated in weak interactions between quarks. Theories suggest that the breaking

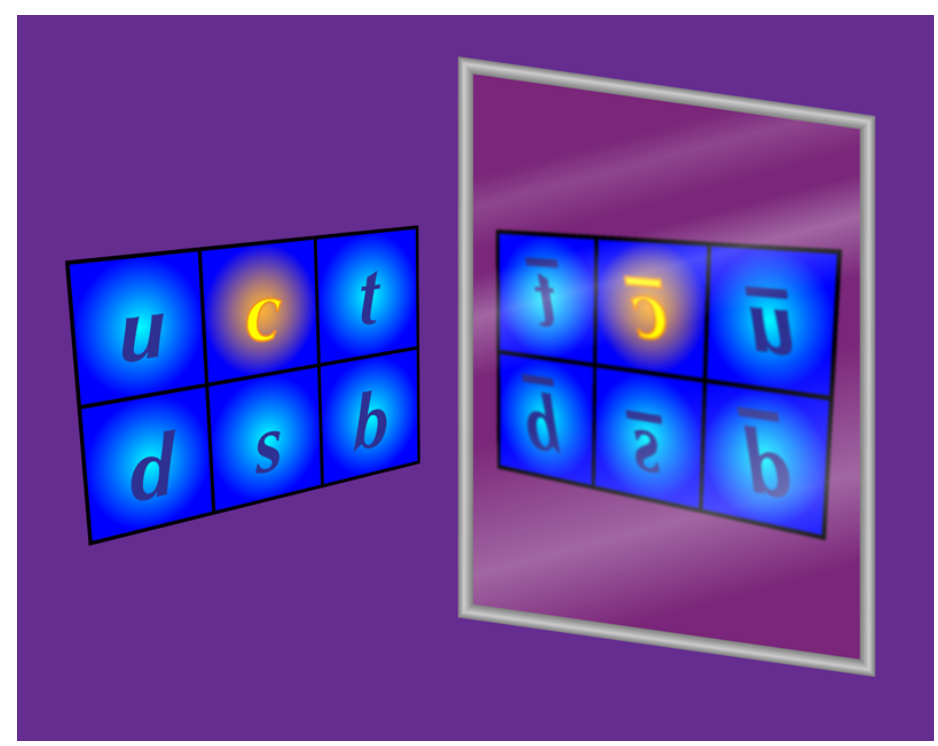

Figure 1: New results from the LHCb experiment have identified a difference in the way charm quarks $(c)$ behave as compared to anticharm quarks $(\bar{c})$, when a spatial inversion (mirror reflection) is taken into account. This so-called charge-parity $(C P)$ violation had previously been observed in strange $(s)$ and beauty $(b)$ quarks. (APS/Alan Stonebraker)

$\ddagger$ Department of Physics, University of Tokyo, Tokyo, Japan of $C P$ symmetry in weak interactions could have favored the creation of matter over antimatter in the early Universe. Experiments have verified $C P$ violation in mesons containing strange and beauty (bottom) quarks [1-3], but the violation in the charm sector has proven harder to measure. After decades of experimental searches, the $\mathrm{LHCb}$ experiment at CERN has finally succeeded in observing a small (few parts in $10^{3}$ ) effect of $C P$ violation in charm decays [4]. This observation is consistent with standard model predictions, but further study of $C P$ violation in the charm sector may reveal new physics beyond the standard model.

In the formalism of quantum physics, $C P$ is an operator that transforms a particle into an antiparticle while simultaneously performing an inversion of spatial variables. If an interaction or a decay remains the same after a $C P$ transformation, then it is said to be $C P$ symmetric. Among the three interactions pertinent to elementary particles, electromagnetic and strong interactions are $C P$ symmetric, while the weak interaction is not.

$C P$ violation in the weak interaction was discovered in 1964 at Brookhaven National Laboratory in New York, where it was shown that the $K_{2}^{0}$ meson, consisting of an antistrange quark and a down quark, decays on rare occasions to two $\pi$ mesons [1]. Such decays require $C P$ symmetry breaking. Later, observations at so-called $B$ factories established $C P$ violation in $B$ mesons (containing beauty quarks) $[2,3]$. To explain these observations, theorists proposed the Cabibbo-Kobayashi-Maskawa (CKM) model, which assumes that the six quarks are "mixed" $[5,6]$ This mixing describes the probability that, for example, a beauty quark will transform through a weak interaction into an up quark. Because of $C P$ violation, this probability will be different than that of an antibeauty quark transforming into an antiup quark.

According to the CKM model (which is part of the standard model), the $C P$ asymmetry in strange quarks is a few times $10^{-3}$, while in beauty quarks it is as large as 0.7 . The predicted $C P$ violation is significantly smaller for charm quarks, with asymmetries of the order of $10^{-4}$ to $10^{-3}$. Researchers have looked for $C P$ violation in the charm sector by studying $D$ mesons, which are the lightest particles containing charm quarks. Because the expected asymmetry is so small, a large number of $D$ and anti- $D$ mesons needs to 
be investigated. The Large Hadron Collider (LHC) at CERN is a proton-proton collider with unprecedented capability to produce massive amounts of $D$ and anti- $D$ mesons. The $\mathrm{LHCb}$ detector, mounted in one of the LHC experimental halls, weighs about 4500 tons and is specifically designed for the study of particles containing charm or beauty quarks [7]. Its components gather information about the identity, trajectory, momentum, and energy of each particle produced by high-energy proton-proton collisions, and the detector as a whole can single out individual particles from the billions that spray out from the collision point.

Equal numbers of neutral $D\left(D^{0}\right)$ and antineutral $D$ mesons $\left(\bar{D}^{0}\right)$ are produced either as primary products at a proton-proton collision or as secondary products from $B$ meson decays. The $\mathrm{LHCb}$ has a superb capability to distinguish $D^{0}$ from $\bar{D}^{0}$ in a particular event based on the information of the accompanying particles. The experiment accumulated a large data sample and extracted approximately 53 million $D \rightarrow K^{-} K^{+}$decays, which includes both $D^{0}$ and $\overline{D^{0}}$ decaying to charged $K$ meson pairs. In addition, the data contained 17 million $D \rightarrow \pi^{-} \pi^{+}$decays, which again could either be $D^{0}$ or $\bar{D}^{0}$ decaying to charged $\pi$ meson pairs [4].

$C P$ asymmetry in each decay is given by the fractional asymmetry between the number of $D^{0}$ mesons decaying to $K^{-} K^{+}\left(\pi^{-} \pi^{+}\right)$pairs and that of $\bar{D}^{0}$ to $K^{-} K^{+}\left(\pi^{-} \pi^{+}\right)$pairs: $A(f)=\frac{N\left(D^{0} \rightarrow f\right)-N\left(\bar{D}^{0} \rightarrow f\right)}{N\left(D^{0} \rightarrow f\right)+N\left(\bar{D}^{0} \rightarrow f\right)}$, where $f=K^{-} K^{+}$or $\pi^{-} \pi^{+}$. In order to cancel small non-CP contributions that are common to both $K^{-} K^{+}$and $\pi^{-} \pi^{+}$decays, such as asymmetries in detection efficiencies and particle production mechanism in proton-proton collisions, the experiment measures the difference of the $C P$ asymmetries in $D \rightarrow K^{-} K^{+}$and $D \rightarrow$ $\pi^{-} \pi^{+}$decays. The $\mathrm{LHCb}$ experiment has obtained $\Delta A_{C P}=$ $A\left(K^{-} K^{+}\right)-A\left(\pi^{-} \pi^{+}\right)=(-1.54 \pm 0.29) \times 10^{-3}$. The result is 5.3 standard deviations from zero (or no asymmetry), which clearly establishes $C P$ violation in the decay of charm mesons.

$C P$ violation in the quark system has long been established for the strange and beauty quarks, which belong to the category of quarks (along with the down quark) that have a charge of $-1 / 3 e$, where $e$ is the elementary charge. The LHCb result confirms, for the first time, $C P$ asymmetry for the charm quark that belongs to the category of quarks (along with up and top) having a charge of $+2 / 3 e$. The obtained result lies at the upper end of the standard model expectations, $10^{-4}$ to $10^{-3}$. Could this slightly large measurement be hinting at new physics? Theorists have shown that the amount of $C P$ violation in all the quarks (as predicted by the CKM model) is not enough to explain matter dominance in our Universe. The implication is that the stan- dard model is not a complete theory and that still unknown particles and interactions await to be discovered.

Many of the theoretical models beyond the standard model result in enhancement of $C P$ violation for the $+2 / 3 e$ charge quark system. And here the smallness of $C P$ asymmetry in charm quarks could become an asset. If, for example, an interaction outside of the standard model increased the $C P$ asymmetry by $10^{-3}$, then that would be more noticeable in charm quarks than in strange or beauty quarks, for which the standard model $C P$ violation is relatively large [8]. The $\mathrm{LHCb}$ result with the current precision cannot yet tell if unknown particles and/or interactions are affecting the $C P$ violation in the charm sector. But as more data is collected, the charm quark system could serve as a tool to probe physics beyond the standard model.

The charm quark has a history of upending particle physics. In 1974, the discovery of the bound state of charm and anticharm quarks, the $J / \psi$ particle, revolutionized our understanding of the subatomic world $[9,10]$. Now this quark may be coming back to play a role in uncovering the mystery of the lopsided Universe.

This research is published in Physical Review Letters.

\section{REFERENCES}

[1] J. H. Christenson, J. W. Cronin, V. L. Fitch, R. Turlay et al., "Evidence for the $2 \pi$ decay of the $K_{2}^{0}$ meson," Phys. Rev. Lett. 13, 138 (1964).

[2] B. Aubert et al. (BABAR Collaboration), "Observation of $C P$ violation in the $B^{0}$ meson system," Phys. Rev. Lett. 87, 091801 (2001).

[3] K. Abe et al. (Belle Collaboration), "Observation of large $C P$ violation in the neutral $B$ meson system," Phys. Rev. Lett. 87, 091802 (2001).

[4] R. Aaij et al. (LHCb Collaboration), "Observation of $C P$ violation in charm decays," Phys. Rev. Lett. 122, 211803 (2019).

[5] N. Cabibbo, "Unitary symmetry and leptonic decays," Phys. Rev. Lett. 10, 531 (1963).

[6] M. Kobayashi and T. Maskawa, " $C P$-violation in the renormalizable theory of weak interaction," Prog. Theor. Phys. 49, 652 (1973).

[7] A. A. Alves Jr. et al. (LHCb Collaboration), "The LHCb detector at the LHC," J. Inst. 3, S08005 (2008).

[8] Y. Grossman, A. L. Kagan, and Y. Nir, "New physics and $C P$ violation in singly Cabibbo suppressed $D$ decays," Phys. Rev. D 75, 036008 (2007).

[9] J. J. Aubert et al., "Experimental observation of a heavy particle J," Phys. Rev. Lett. 33, 1404 (1974).

[10] J. -E. Augustin et al., "Discovery of a narrow resonance in $e^{+} e^{-}$ annihilation," Phys. Rev. Lett. 33, 1406 (1974).

10.1103/Physics. 12.52 